Arq. Bras. Med. Vet. Zootec., v.62, n.4, p.801-805, 2010

\title{
Obstrução intestinal por Pythium insidiosum em um cão: relato de caso
}

\author{
[Intestinal obstruction by Pythium insidiosum in a dog: case report] \\ P.S. Hunning, G. Rigon, C.S. Faraco, S.P. Pavarini, D. Sampaio, W. Beheregaray, D. Driemeier \\ Faculdade de Veterinária - UFRGS \\ Porto Alegre, RS
}

\begin{abstract}
RESUMO
Relata-se um caso de pitiose intestinal em um Husky Siberiano, de um ano de idade, macho atendido em um Hospital Veterinário Escola com sinais de obstrução intestinal. Ao exame físico, notou-se massa cilíndrica na região abdominal cranial, posteriormente confirmada por meio de radiografia e ultrassonografia. Durante a laparotomia exploratória, constatou-se massa extraluminal envolvendo o jejuno e alterações na parede do órgão. Realizou-se a ressecção da porção afetada do intestino e, posteriormente, anastomose. $\mathrm{O}$ exame histológico do tecido evidenciou inflamação piogranulomatosa acentuada. Na coloração de prata metenamina de Grocott, hifas septadas foram observadas. O diagnóstico de infecção por Pythium insidiosum foi confirmado por meio da imunoistoquímica. Após a cirurgia, o animal restabeleceu a defecação; no $30^{\circ}$ dia pós-cirúrgico, foi relatada ainda presença de diarreia. $\mathrm{O}$ tratamento com itraconazol e terbinafina foi instituído durante 60 dias. Após dois anos do procedimento cirúrgico e do tratamento com antifúngicos orais, o cão não apresentou recidiva.
\end{abstract}

Palavras-chave: cão, pitiose intestinal, imunoistoquímica

\begin{abstract}
A case of intestinal pythiosis in a one-year-old male Siberian Husky treated at a Veterinary School Hospital with signs of intestinal obstruction is reported. At physical examination, a cylindrical mass was palpable in the cranial abdomen, later confirmed by radiography and ultrasonography. During the exploratory laparotomy, it was evidenced an extraluminal mass involving the jejunum and alterations of the wall in the organ. After that, a resection of the affected portion of the intestine was made followed by anastomosis. The histologic examination evidenced accented pyogranulomatous inflammation. By the Grocott methenamine silver stain, branching hyphae were observed. The diagnosis of infection by Pythium insidiosum was confirmed by immunohistochemistry. The animal reestablished the defecation after the surgery; on the $30^{\text {th }}$ postoperative day, it was also reported the presence of diarrhea. The treatment with itraconazol and terbinafina was instituted for 60 days. Two years after the surgical procedure and treatment with oral antifungals, the dog did not show recurrence.
\end{abstract}

Keywords: dog, intestinal pythiosis, immunohistochemistry

\section{INTRODUÇÃO}

A pitiose é uma doença piogranulomatosa crônica, causada por oomicetos pertencentes ao reino Straminipila, do gênero Pythium (Cock et al., 1987; Graham et al., 2000; Rech et al., 2004; Rodrigues et al., 2006), e mais de 120 espécies são encontradas em plantas e peixes, podendo ocasionar doenças (Leal et al., 2001; Santurio et al., 2006). Somente o P. insisdiosum acomete mamíferos, entre os quais equinos, caninos, felinos, bovinos e humanos. Nas espécies não domésticas, já foi citado em um urso cativo, um jaguar e um camelo (Camus et al., 2004; Wellehan et al., 2004). A doença ocorre principalmente em regiões de clima tropical, subtropical e temperado, e já foi relatada no continente americano, alguns países europeus,

Recebido em 20 de novembro de 2009

Aceito em 2 de agosto de 2010

E-mail: paulastieven@yahoo.com.br 
sudeste asiático, Oceania e, mais recentemente, na África (Rivierre et al., 2005; Santurio et al., 2006). O calor e a umidade são fatores necessários para o desenvolvimento do agente, que ocorre principalmente durante o verão e o outono. A forma infectante são os zoósporos móveis, que se desenvolvem em regiões alagadiças, apresentam quimiotaxia por pelos e são capazes de penetrar em soluções de continuidade da pele e das mucosas (Santurio et al., 2006).

A espécie equina é a mais atingida pela pitiose, e não há predisposição quanto à raça, sexo ou idade. As lesões cutâneas são as mais frequentes e atingem, principalmente, as extremidades distais dos membros e a porção ventral da parede toracoabdominal, provavelmente devido ao maior tempo de contato com águas contaminadas por zoósporos (Santurio et al., 2006). A forma intestinal é a segunda mais frequente da infecção pelo $P$. insidiosum em equinos e cursa com episódios de cólica, causada pela presença de massas teciduais, levando à redução e/ou obstrução do lúmen intestinal (Allison e Gillis, 1990)

Os caninos são a segunda espécie mais atingida pela pitiose, que acomete primariamente a pele e o trato gastrintestinal (Fischer et al., 1994; Rech et al., 2004; Santurio et al., 2006). A forma gastrintestinal acomete principalmente machos jovens, de grande porte, e se manifesta com vômito, diarreia esporádica, perda de peso progressivo e dor abdominal (Miller, 1985; Rech et al., 2004). A formação de massas palpáveis, semelhantes a tumorações no trato gastrintestinal, é comum (Leal et al., 2001; Santurio et al., 2006). Os tratamentos descritos incluem cirurgia, uso de antifúngicos e imunoterapia (Leal et al., 2001; Santurio et al., 2006). Destes, a cirurgia de retirada dos tecidos afetados, com ampla margem de segurança, é a única forma efetiva de tratamento (Santurio et al., 2006).

O diagnóstico definitivo da infecção por $P$. insidiosum deve ser realizado por cultura seguida de identificação do patógeno, baseando-se em características culturais, morfológicas e reprodutivas. Quando a cultura não for possível, técnicas de sorologia, imunoistoquímica e PCR podem ser utilizadas (Racich et al., 2005).
Este trabalho teve o objetivo de relatar um caso de pitiose intestinal em um cão atendido em um Hospital Veterinário Escola.

\section{CASUística}

Um cão da raça Husky Siberiano, macho, um ano de idade, com aproximadamente $17 \mathrm{~kg}$, foi atendido no Hospital de Clínicas Veterinárias da Universidade Federal do Rio Grande do Sul por apresentar disquesia e êmese. Apresentava também anorexia e emagrecimento progressivo. $\mathrm{O}$ animal tinha acesso à região rural, onde havia um riacho. Ao exame físico era possível a palpação de massa cilíndrica medindo aproximadamente $7 \times 4 \mathrm{~cm}$ na porção abdominal cranial, cuja presença no intestino delgado foi confirmada pelo exame radiográfico. Ao exame ultrassonográfico do abdômen, constatou-se aumento localizado e acentuado da parede do intestino delgado com aspecto hipoecogênico. $\mathrm{O}$ cão foi encaminhado para a laparotomia exploratória.

Como medicação pré-anestésica, utilizou-se o sulfato de morfina $(0,5 \mathrm{mg} / \mathrm{kg}, \mathrm{IM})$. A indução foi realizada com propofol $(4 \mathrm{mg} / \mathrm{kg}$, IV), e a manutenção com isoflurano vaporizado em oxigenação a $100 \%$ com circuito semiaberto. Após a abertura do abdômen, evidenciou-se uma grande massa extraluminal no jejuno, provocando estreitamento da luz intestinal. Notou-se também que a parede do intestino adjacente encontrava-se espessada e os linfonodos mesentéricos, aumentados. Em vista disso, em torno de $30 \mathrm{~cm}$ de intestino delgado foram removidos. Em seguida, realizou-se a anastomose, sutura do tecido muscular, do subcutâneo e da pele. O segmento removido foi aberto, evidenciando trajetos necróticos amarelados, em meio a tecido fibroso claro (Fig. 1).

Para o pós-operatório, utilizou-se tramadol $(2 \mathrm{mg} / \mathrm{kg}$, IM, TID) durante dois dias, metronidazol $(10 \mathrm{mg} / \mathrm{kg}$, VO, TID) durante 10 dias e cefalexina $(30 \mathrm{mg} / \mathrm{kg}, \mathrm{VO}$, BID) pelo mesmo período. Durante dois dias, o animal permaneceu em jejum associado a fluidoterapia, com glicose a $50 \%$ e frutose diluídas em solução de $\mathrm{NaCl} 0,9 \%$. No terceiro dia, foi instituído o uso de ração pastosa, que se prolongou por mais quatro dias e, posteriormente, o de ração 
comercial especial para animais com distúrbios intestinais.

O fragmento de intestino, removido cirurgicamente e encaminhado ao setor de patologia veterinária, foi fixado em solução de formalina a $10 \%$. O fragmento de intestino foi processado rotineiramente para exame histopatológico, foi incluído em parafina, cortado a $5 \mu \mathrm{m}$ de espessura e corado pela hematoxilina e eosina (HE) e coloração de prata metenamina de Grocott (GMS). Cortes foram submetidos ao exame imunoistoquímico utilizando a técnica de streptavidina-biotina ligada à peroxidase. Anticorpo policlonal anti-P.insidiosum produzido em coelhos foi empregado à diluição de 1:100 em phosphate buffered saline (PBS). Utilizou-se, como recuperador antigênico, tripsina e, como cromógeno, tetra-hidrocloreto de 3,5-diaminobenzidina (DAB-DAKO). Foram inseridos controles positivos de $P$. insidiosum, confirmado com isolamento; como controle negativo, foi incluído um caso de infecção por Conidiobolus spp.

Ao exame histopatológico, havia proliferação de tecido conjuntivo fibroso associado a um processo inflamatório transmural piogranulomatoso multifocal intenso, caracterizado por grande quantidade de eosinófilos, macrófagos e, em menor número, neutrófilos, plasmócitos e células gigantes. Observou-se necrose caseosa multifocal contendo estruturas semelhantes a hifas, algumas septadas e/ou ramificadas, negativamente coradas pelo HE e evidenciadas pela GMS. Estas revelaram marcação positiva quando submetidas à imunoistoquímica para P.insidiosum (Fig. 2).

Ao $10^{\circ}$ dia pós-operatório, o animal retornou para revisão, e foi instituída a terapia com itraconazol $(5 \mathrm{mg} / \mathrm{kg}, \mathrm{VO}$, BID) associada à terbinafina $(10 \mathrm{mg} / \mathrm{kg}, \mathrm{VO}, \mathrm{SID})$ durante 30 dias. Ao $30^{\circ}$ dia de tratamento, a proprietária relatou que o animal se encontrava em estado alerta, com apetite normal e sem vômitos, no entanto ainda apresentava diarreia. $\mathrm{O}$ exame hematológico revelou eosinofilia leve, 1462 células $/ \mu \mathrm{L}$, e monocitopenia, 86 células $/ \mu \mathrm{L}$. O perfil bioquímico do plasma e a ultrassonografia não apresentaram alterações. O tratamento foi recomendado por mais 30 dias. Após dois anos do procedimento cirúrgico e do tratamento com antifúngicos orais, o cão não apresentou sinais de recidiva.

\section{DISCUSSÃO E CONCLUSÕES}

A pitiose gastrintestinal é uma doença crônica, cuja forma de infecção pode ser através de penetração pela pele ou ingestão de água contaminada (Grooters, 2002). Neste relato, a infecção provavelmente ocorreu pelo acesso que o cão tinha ao riacho. $\mathrm{O}$ caso foi recebido em abril, ou seja, outono. Entretanto, como o crescimento do fungo é lento, cerca de dois a sete meses, é provável que o cão tenha se infectado na primavera ou no verão, quando o calor juntamente com as chuvas propiciam $\mathrm{O}$ crescimento do agente, tornando as infecções por Pythium mais frequentes (Rech et al., 2004). A característica epidemiológica e o fato de ser um animal jovem e de grande porte são descritos como predisponentes à pitiose gastrintestinal em caninos (Miller, 1985).

Os profissionais que atendem animais que apresentem perda de peso, vômito, diarreia e massas palpáveis em abdômen, como este cão, devem incluir a pitiose intestinal no diagnóstico diferencial (Miller, 1985). Como houve suspeita inicial de ser um processo neoplásico, o material foi fixado em solução de formalina $10 \%$, o que, dessa forma, inviabilizou o cultivo, o isolamento do agente e a indução da zoosporangênese, que, associados aos achados histopatológicos, são os métodos rotineiramente utilizados para a confirmação da etiologia da doença (Santurio et al., 2006). Como houve suspeita de linfoma, não foi coletado material para cultivo. Quando isso ocorre, somente o exame histopatológico não é confirmatório da pitiose, pois existem outros fungos que podem produzir o mesmo tipo de lesão, como as zigomicoses e as lagenidioses (Racich et al., 2005). O emprego da imunoistoquímica é vantajoso, pois pode ser aplicado sobre tecidos parafinados, permitindo o diagnóstico quando a cultura ou o soro não estão disponíveis (Grooters, 2002). Nesse caso, a técnica da imunoistoquímica foi de grande importância para confirmação da doença e diagnóstico específico. 


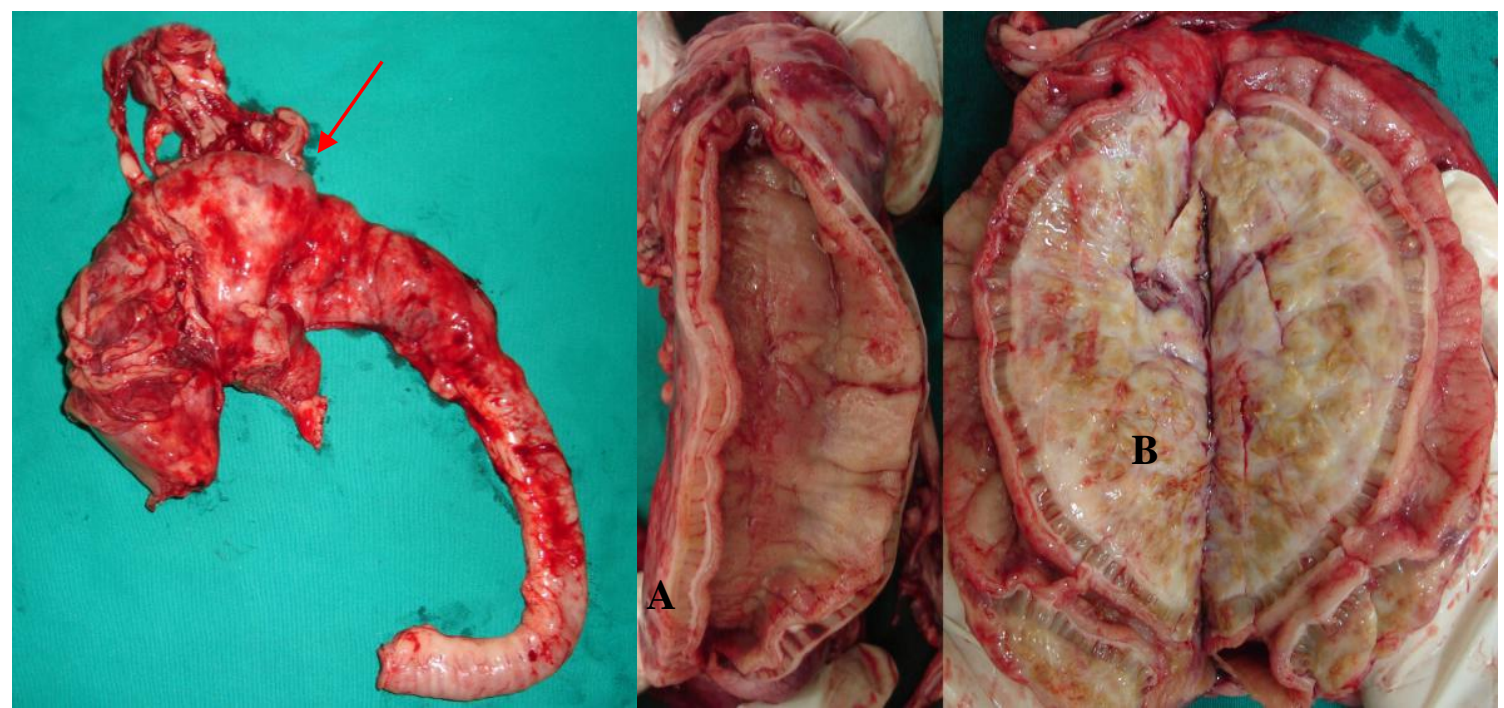

Figura 1. Cão: segmento do intestino delgado removido. Aspecto macroscópico da porção externa do intestino $(\rightarrow)$. Aspecto macroscópico da porção interna do intestino: corte longitudinal, evidenciando espessamento intestinal (A) e interior da massa caseosa (B).

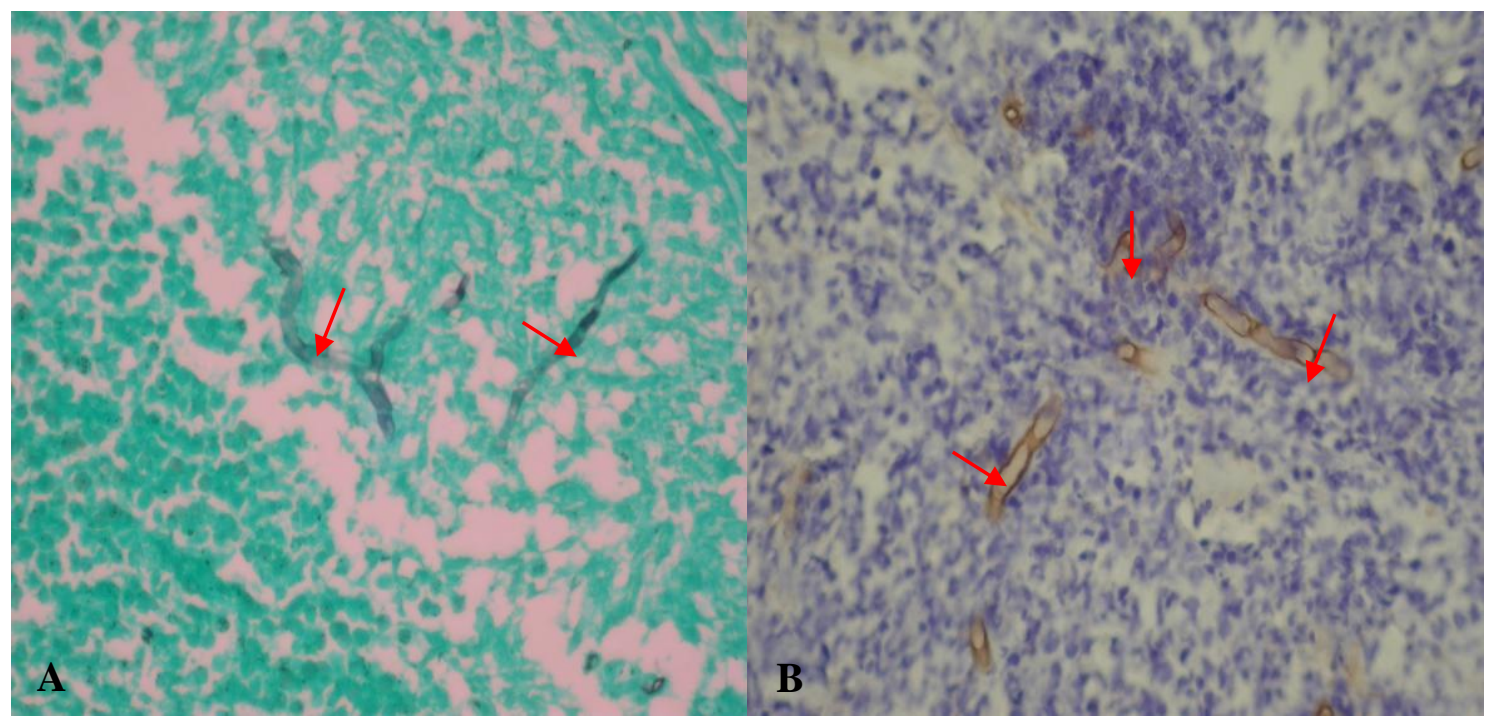

Figura 2. Cão: (A) aspecto microscópico das hifas coradas pela coloração de GMS ( $\rightarrow$ ). Obj 40. (B) marcação positiva na imunoistoquímica com anticorpo policlonal anti-Pythium insidiosum $(\rightarrow)$. Obj 40.

O aumento da espessura da parede intestinal frequentemente resulta em obstrução parcial (Liljebjelke et al., 2002; Racich et al., 2005). Neste caso, a obstrução intestinal foi acarretada pelo crescimento de um nódulo inflamatório que se estendia a partir da serosa, com consequente compressão da luz intestinal. A pitiose apresentase como uma doença infiltrativa, frequentemente localizada no estômago e intestino delgado e ocasionalmente envolvendo outros tecidos (Miller, 1985; Rech et al., 2004). Além do jejuno, os linfonodos mesentéricos também se encontravam alterados.

Os achados histopatológicos descritos neste trabalho, de reação inflamatória 
piogranulomatosa tipicamente eosinofílica condizem com a literatura (Fischer et al., 1984; Miller, 1985; Bentinck-Smith et al., 1989; Rech et al., 2004; Racich et al., 2005).

A escolha da ressecção cirúrgica, no momento da laparotomia exploratória, é o tratamento mais eficaz. A associação de itraconazol a terbinafina para o pós-operatório foi utilizada como medida preventiva para o crescimento do agente, baseando-se no fato de esses agentes possuírem efeito sinérgico quando utilizados em conjunto e terem sido usados com algum índice de sucesso (Racich et al., 2005; Santurio et al., 2006).

Os dados epidemiológicos, os sinais clínicos e os achados histopatológicos deste relato são similares aos descritos na literatura sobre a pitiose intestinal em cães, sendo sua confirmação possível pela imunoistoquímica.

\section{REFERÊNCIA BIBLIOGRÁFICA}

ALLISON, N.; GILLIS, J.P. Enteric pythiosis in a horse. J. Am. Vet. Med. Assoc., v.196, p.462464, 1990.

BENTINCK-SMITH， J.; PADHYE， A.A.; MASLIN, W.R. et al. Canine pythiosis isolation and identification of Pythium insidiosum. J. Vet. Diagn. Invest., v.1, p.295-298, 1989.

CAMUS, A.C.; GROOTERS, A.M.; AQUILAR, R.E. Granulomatous pneumonia caused by Pythium insidiosum in a central American jaguar, Panthera onca. J. Vet. Diagn. Invest., v.16, p.567-571, 2004.

COCK, A.W.A.M.; MENDOZA, L.; PADHYE, A.A. et al. Pythium insidiosum sp. nov., the etiologic agent of pythiosis. J. Clin. Microbiol., v.25, p.344-349, 1987.

FISCHER, J.R.; PACE, L.W.; TURK, J.R. et al. Gatrointestinal pythiosis in Missouri dogs: eleven cases. J. Vet. Diagn. Invest., v.6, p.380$382,1994$.
GRAHAM, J.P.; NEWELL, S.M.; ROBERTS, G.D. et al. Ultrasonographic features of canine gastrointestinal pythiosis. Vet. Rad. Ultrasound, v.41, p.273-277, 2000

GROOTERS, A.M. New diagnostic tests for pythiosis, 2002. Disponível em: <http://rottweilerhealth.org/pdfs/july_pythiosis_g rooters_02.pdf>. Acessado em: 01 mai. 2007.

LEAL, A.T.; LEAL, A.B.M.; FLORES, E.F. et al. Pitiose. Cienc. Rural, v.31, p.735-743, 2001.

LILJEBJELKE， K.A.; ABRAMSON， C.; BROCKUS, $\mathrm{C}$ et al. Duodenal obstruction caused by infection with Pythium insidiosum in a 12-week-old puppy. J. Am. Vet. Med. Assoc., v.220, p.1188-1191, 2002.

MILLER, R.I. Gastrointestinal phycomicosis in 63 dogs. J. Am. Vet. Med. Assoc., v.186, p.473478, 1985.

RACICH, P.M.; GROOTERS, A.M; TANG, K.N. Gatrointestinal pythiosis in two cats. J. Vet. Diagn. Invest., v.17, p.262-269, 2005.

RECH, R.R.; GRAÇA, D.L.; BARROS, C.S.L. Pitiose em um cão: relato de caso e diagnósticos diferenciais. Clin. Vet., v.90, p.68-72, 2004.

RIVIERRE, C.; LAPRIE, C.; GUIARDMARIGNY, O. et al. Pythiosis in Africa. Emerg. Infect. Dis., v.11, p.479-481, 2005.

RODRIGUES, A.; GRAÇA, D.L.; FONTOURA, C. et al. Intestinal dog pythiosis in Brazil. $J$. Mycol. Med., v.16, p.37-41, 2006.

SANTURIO, J.M.; ALVES, S.H.; PEREIRA, D.B. et al. Pitiose: uma micose emergente. Acta Scient. Vet., v.34, p.1-14, 2006.

WELLEHAN, J.F.; FARINA, L.L.; KEOUGHAN, C.G. et al. Pythiosis in a dromedary camel (Camelus dromedarius). J. Zoo Wildl. Med., v.35, p.564-568, 2004. 